\title{
The Influence of Right Ventricular Afterload in Cardiac Resynchronization Therapy: A CircAdapt Study
}

\author{
Clemens Zeile ${ }^{1}$, Thomas Rauwolf ${ }^{2}$, Alexander Schmeisser ${ }^{2}$, Tobias Weber $^{1}$, Sebastian Sager ${ }^{1}$ \\ ${ }^{1}$ Institute for Mathematical Optimization, Otto-v.-Guericke-University, Magdeburg, Germany \\ ${ }^{2}$ Department of Cardiology, Otto-v.-Guericke-University, Magdeburg, Germany
}

\begin{abstract}
The individual response to cardiac resynchronization therapy $(C R T)$ remains difficult to predict, and some research has focused on right ventricle $(R V)$ dysfunction parameters in an effort to shed light on this problem. In a recent clinical study, researchers investigated the interaction of the $R V$ with the pulmonary system. In this article, we analyze this issue by performing a computer simulation with the established CircAdapt model. Our results indicate that $R V$ afterload at baseline is highly correlated with positive acute CRT response in terms of positive stroke volume $(S V)$ and $R V$ ejection fraction $(E F)$ change.
\end{abstract}

\section{Introduction}

CRT is a widely accepted and successful treatment for heart failure (HF) occurring in combination with electrical conduction delay, i.e. left bundle branch block (LBBB). Large clinical studies have shown that CRT improves left venctricular (LV) function and affects long-term survival. However, the response of any given individual to CRT response is difficult to predict for reasons that are not completely understood. Analysis of RV fractional area change (FAC) and tricuspid annular plane excursion have shown that right ventricle (RV) function is an important predictor of echocardiographic and clinical outcomes following CRT. Studies of the relationship of these non-invasive parameters to CRT response have yielded conflicting results, however [1]. To clarify this issue, a recent study [2] of invasive criteria measured $\mathrm{RV}$ pressure volume (PV) loops and concluded that RV-pulmonary arterial coupling ratio specifically, RV afterload - can indeed predict a given individual's response to CRT.

Several studies have elaborated the acute CRT response due to various conditions via the multipatch module of CircAdapt [3,4], a well-established tool that allows rapid simulation of the heart and circulatory system [5,6]. Recently, CircAdapt was also used in this context to test both mechanical dyssynchrony and RV contractility [7]. In this article, we also use CirAdapt to examine in silico the results of the aforementioned study. We first simulate RV afterload variations in terms of pulmonary hypertension $(\mathrm{PH})$, increased arterial stiffness, and dilated cardiomyopathy (DCM) - all based on an HF setting. Then, we conduct a specific simulation for two representative patients. In both cases, we evaluate the acute CRT response.

\section{Methods}

\subsection{Patient data}

Magdeburger cardiologists included 111 patients with standard CRT indications in their study. Data collection was performed via echocardiography, as well as leftand right-heart catheterization including RV conductance catheterization (PV loop measurement). Table 2.1 gives an extract of mean patient data (response defined as LV end systolic volume (LVESV) remodeling $\geq 15 \%$ after six months). See [2] for further details.

\begin{tabular}{lcc}
\hline \hline & Responder & Non-responder \\
\hline LV EF (\%) & 33 & 29 \\
LV EDP (mmHg) & 19 & 24 \\
LV ESP (mmHg) & 131 & 123 \\
RV EF (\%) & 47 & 39 \\
RV EDP (mmHg) & 7 & 9 \\
RV ESP (mmHg) & 27 & 42 \\
CO (1) & 4.8 & 4.3 \\
Ea (mmHg/ml) & 0.38 & 0.62 \\
Ees (mmHg/ml) & 0.35 & 0.34 \\
PA-C (ml/mmHg) & 3.3 & 2.1 \\
PVR (dyn) & 137 & 195 \\
\hline \hline
\end{tabular}

Table 1. Study data, with end-diastolic pressure (EDP), end-systolic pressure (ESP), cardiac output (CO), arterial elastance (Ea), end-systolic elastance (Ees), pulmonary artery compliance (PA-C), pulmonary vascular resistance (PVR). 


\subsection{CircAdapt and simulation of $\mathrm{HF}$ with CRT}

The CircAdapt model consists of several modules that permit realistic simulation of cardiovascular mechanics and hemodynamics. It features 29 ordinary differential equations, and thus justifies the label 'phenomological'. For brevity's sake, we skip the details and refer the interested reader instead to $[3,4]$. Using the already established algorithm, we simulated HF with LBBB for our mean responder patient. Using a reference simulation, we incorporated a heart rate of 71 beats/min and adjusted blood volume and mean aortic pressure to meet both $\mathrm{CO}$ as well as LV and RV volume data. As in [6], we applied adaptation protocols before inducing HF. This involved decreasing contractility and dividing the ventricles into patches with LBBB-like atrioventricular $(220 \mathrm{~ms})$ and intraventricular $(135 \mathrm{~ms})$ activation delays. We then varied the RV afterload by means of three simulations - pulmonary artery stiffness, DCM, and PH. We also examined increased and decreased contractility. For the contractility and DCM simulations, we changed the right ventricular parameters $A_{\mathrm{m}, \mathrm{Ref}}, \sigma_{\mathrm{f}, \mathrm{Act}}$. Similarly, we adjusted the nonlinear stiffness exponent $k$ and PH parameter $p_{0 \mathrm{AV}}$ to meet different pressure conditions. This resulted in a total of about 80 combinations, for each of which we applied a homoestatic pressure-flow regulation algorithm [5] before performing the CRT ventricular activation pattern from [6]. The acute CRT response was observed by running 20 heart cycles without pressureflow adaptation. To consider RV afterload independently of other factors, LV dysfunction was kept constant for all simulations.

\subsection{Afterload evaluation}

For brevity we skip units in this section. The wave impedance $\mathrm{R}_{\text {wave }}$ and the cross-sectional compliance at the arterial entrance $C_{\text {art }}$ were deduced in [8] as a priori model parameters:

$\mathrm{R}_{\mathrm{wave}}=\sqrt{\frac{\rho(k / 3-1) p_{\mathrm{art}}}{A_{c}\left(A_{c}+0.5 \cdot A_{w}\right)}}, \quad C_{\mathrm{art}}=\frac{L_{c}\left(A_{c}+0.5 \cdot A_{w}\right)}{(k / 3-1) p_{\mathrm{art}}}$,

where $\rho$ is the blood density, $p_{\text {art }}$ is the PA pressure, and $A_{c}$ and $A_{w}$ are the cross-sectional pulmonary cavity and wall area, respectively. A posteriori, we decided to rely on clinical driven afterload criteria: mean PA pressure $P A P_{\text {mean }}$, together with

$$
\begin{aligned}
\mathrm{TPG} & =\operatorname{mean}\left(p_{\text {art }}-p_{\mathrm{LA}}\right), & \mathrm{Puls} & =\frac{\max A_{c}-\min A_{c}}{\min A_{c} / 100}, \\
\mathrm{PVR} & =\frac{80 \cdot T P G}{C O}, & \mathrm{PA}-\mathrm{C} & =\frac{S V}{\max p_{\text {art }}-\min p_{\text {art }}}, \\
E_{a} & =\frac{E S P}{S V}, & \mathrm{Cap} & =\frac{\max A_{c}-\min A_{c}}{\max p_{\text {art }}-\min p_{\text {art }}},
\end{aligned}
$$

where Puls denotes the pulsatility, Cap is the capacitance, and TPG is the transpulmonary gradient. In this study, we calculated ESP as peak ventricular pressure divided by volume.

\subsection{Patient specification}

We performed a global sensitivity analysis to identify sensitive right ventricular volume parameters. On the basis of the obtained insights, we were able to manually adapt the parameters $A_{m, r e f}, V_{\mathrm{Wall}}, T R, \sigma_{\mathrm{f}, \mathrm{Act}}, p_{0 \mathrm{AV}}$, and $k$ in order to represent the patient's PV loop data. We selected a typical patient with a triangle-shaped RV-PV loop and another patient with a reverse triangle curved RV-PV loop. Triangle-shaped loops indicate low afterload and, therefore, a high likelihood of CRT response; reverse triangular curved loops imply the opposite. The previously-described CRT algorithm was then performed for both cases.

\section{Results}

After electrical synchronization is induced, an immediate improvement in SV and RV EF generally appears in the simulated heart. The relationship between the change in RV afterload and the change in SV and RV EF is illustrated in fig. 1. PH and artery stiffness strongly correlate (negatively) with both criteria. Elevated DCM shows also a negative effect on CRT response, but not as intense as the others. The response behavior to varied contractility, which appears even with a nonlinear correlation to SV and $\mathrm{RV} \mathrm{EF}$, is also depicted for comparison purposes. Altogether, $\Delta \mathrm{SV}$ is between $-10.8 \%$ and $8.4 \%$, while $\Delta \mathrm{RV} E \mathrm{EF}$ is between $-11.2 \%$ and $10.3 \%$. For all four parameter groups, the trend of SV change is in line with the trend of RV EF.

Table 2 summarizes computed Pearson correlation coefficients for the introduced afterload criteria at baseline to $\Delta \mathrm{SV}$ and $\Delta \mathrm{RV} \mathrm{EF}$. The strongest (negative) correlation appears for $E_{a}$, which confirms the results in [2]. The a priori indicators $\mathrm{R}_{\mathrm{wave}}$, and $C_{\text {art }}$ predict slight CRT changes. Changes of afterload criteria from pre- to post-CRT are also analyzed with respect to $\triangle \mathrm{SV}$ and $\triangle \mathrm{RV} E F$. Interestingly, $P A P_{\text {mean }}$ and TPG correlate positively with both $\triangle \mathrm{SV}$ and $\triangle \mathrm{RV}$ EF. The effect of increasing $P A P_{\text {mean }}$, however, is insignificant compared to the effect of increasing $\mathrm{SV}$, as indicated by the strong association of $\Delta E_{a}$ and $\Delta \mathrm{PVR}$ to $\Delta \mathrm{SV}$.

Figure 2, illustrates simulated patient specific PV loops. Their simulated CRT reaction is shown in table 3, where patient "triangular" outperforms patient "reverse triangular". 

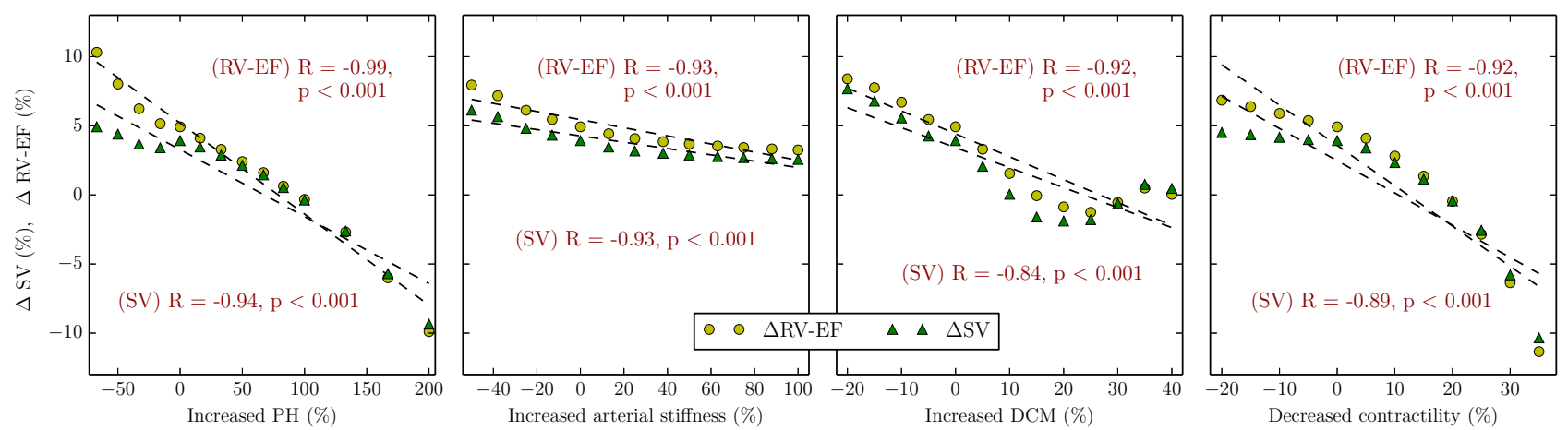

Figure 1. Acute CRT response in relation to RV afterload and contractility parameter variation.

\begin{tabular}{l|cccccccccc} 
& $\mathrm{R}_{\text {wave }}$ & $C_{\text {art }}$ & $P A P_{\text {mean }}$ & $E_{a}$ & ESP & PVR & PA-C & TPG & Puls & Cap \\
\hline R of $\Delta$ SV to baseline AC & -0.01 & 0.27 & -0.77 & -0.82 & -0.81 & -0.73 & 0.31 & -0.72 & 0.52 & 0.26 \\
R of $\Delta$ SV to $\Delta$ AC & -0.46 & -0.45 & 0.9 & -0.97 & 0.49 & -0.88 & 0.67 & 0.82 & -0.21 & 0.61 \\
\hline R of $\Delta$ RV-EF to baseline AC & -0.03 & 0.28 & -0.84 & -0.89 & -0.89 & -0.78 & 0.34 & -0.78 & 0.54 & 0.27 \\
R of $\Delta$ RV-EF to $\Delta$ AC & -0.47 & -0.5 & 0.94 & -0.95 & 0.43 & -0.92 & 0.81 & 0.7 & -0.42 & 0.71 \\
\hline
\end{tabular}

Table 2. Pearson correlation coefficients of SV and RV-EF CRT change to baseline afterload criteria (AC) and CRT changes of afterload criteria, respectively.
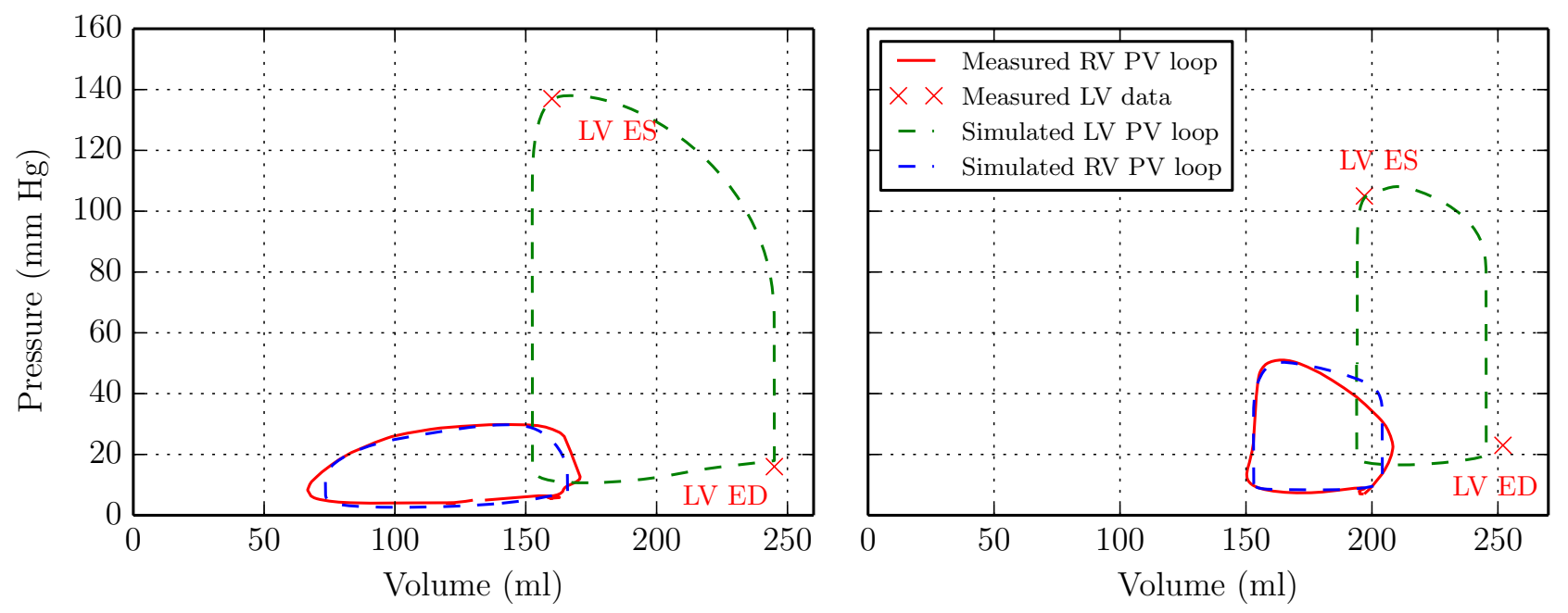

Figure 2. Simulated baseline PV loops, patient with triangular loop (left) and reversed shaped patient (on the right).

\begin{tabular}{l|cccccc|cc} 
& \multicolumn{4}{c}{ Baseline data } & \multicolumn{2}{c}{ Simulated CRT } \\
& LV-EF & RV-EF & SV & RV ESP & PVR & PA-C & $\Delta$ SV & $\Delta$ RV-EF \\
\hline Patient T & $35 \%$ & $48 \%$ & $86 \mathrm{ml}$ & $22 \mathrm{mmHg}$ & $163 \mathrm{dyn}$ & $4.04 \mathrm{ml} / \mathrm{mmHg}$ & $8.2 \%$ & $10.8 \%$ \\
Patient RT & $22 \%$ & $27 \%$ & $55 \mathrm{ml}$ & $57 \mathrm{mmHg}$ & $201 \mathrm{dyn}$ & $1.15 \mathrm{ml} / \mathrm{mmHg}$ & $5.2 \%$ & $7.2 \%$ \\
\hline
\end{tabular}

Table 3. Simulated CRT response with baseline data of triangular (T) and reverse triangular (RT) patient.

\section{Discussion}

The results of this computational study indicate that RV afterload correlates negatively with CRT response. While studies so far [7] focussed on RV function by analyzing contractility, we demonstrate an examination of $\mathrm{PH}$, arterial stiffness and added DCM in the HF-LBBB setting. In 
[7], septal systolic rebound stretch as mechanical dyssynchrony parameter and RV FAC as function parameter were used to elaborate associations with acute CRT response. Our study neglects mechanical dyssynchrony parameters, but deals with clinical parameters and includes also other influences apart from contractility.

Schmeisser et al. [2] identified afterload in terms of $E_{a}$ as main contributing factor to RV PA coupling ratio, so that our in silico analysis is justified. As a first case study, we see afterload as influencing factor - independent of its cause. We evaluated the acute response in terms of $\mathrm{SV}$ change, as experienced in clinical practice. Stolfo et al. [9] found acute RV FAC changes to be a predictor of LVESV long-term remodelling in CRT. In our study, we used RV EF as correlate for RV FAC to interpret acute CRT response in CircAdapt. $\triangle \mathrm{RV}$ EF appeared to be in accordance with $\Delta \mathrm{SV}$ and affirms therefore the hypothesis of negative correlation of RV afterload and CRT success. We conjecture that high RV afterload hinders an increase of SV and therefore also LV remodeling.

\section{Limitations}

In this computational study, we have included only a limited number of the factors affecting RV afterload. The heart, with its two circulatory systems, is very interactive and, for this reason, features such as LV dysfunction have been neglected. The authors in [2]] conjectured that, in many cases, weak LVs were the main cause for low RV pulmonary artery coupling values. Hence, increased RV afterload appeared as secondary effect. Non-responders in the clinical study had larger right ventricles and higher LV dysfunction at baseline. We incorporated this only indirectly into our simulations.

As a phenomenological model, CircAdapt is highly simplified and features only acute CRT response, without capturing the crucial remodeling process. We induced afterload changes solely by means of pressure-flow regulation and did not adapt the system to altered load. Finally, the Pearson correlation coefficient as a statistical tool supports only a very rough and linear notion of relations.

\section{Conclusion}

Computer simulations indicate that RV PV loops (particularly RV afterload) should be carefully considered in making CRT decisions. Future research might focus on a more sophisticated analysis of RV pulmonary-artery coupling factors and causes in LBBB-HF patients.

\section{Acknowledgements}

This project has received funding from the European Research Council (ERC) under the European Unions Hori- zon 2020 research and innovation programme (grant agreement No 647573), which is gratefully acknowledged.

\section{References}

[1] Sharma A, Bax JJ, Vallakati A, Goel S, Lavie CJ, Kassotis J, Mukherjee D, Einstein A, Warrier N, Lazar JM. Metaanalysis of the relation of baseline right ventricular function to response to cardiac resynchronization therapy. The American journal of cardiology 2016;117(8):1315-1321.

[2] Schmeisser A, Rauwolf T, Ghanem A, Groscheck T, Adolf $\mathrm{D}$, Braun-Dullaeus R. Impact of right ventricular-pulmonary interaction on left ventricular remodeling after crt: A right ventricular pressure-volume catheter analysis. Manuscript submitted for publication 2017;

[3] Arts T, Delhaas T, Bovendeerd P, Verbeek X, Prinzen FW. Adaptation to mechanical load determines shape and properties of heart and circulation: the circadapt model. American Journal of Physiology Heart and Circulatory Physiology 2005;288(4):H1943-H1954.

[4] Lumens J, Delhaas T, Kirn B, Arts T. Three-wall segment (triseg) model describing mechanics and hemodynamics of ventricular interaction. Annals of biomedical engineering 2009;37(11):2234-2255.

[5] Walmsley J, Arts T, Derval N, Bordachar P, Cochet H, Ploux S, Prinzen FW, Delhaas T, Lumens J. Fast simulation of mechanical heterogeneity in the electrically asynchronous heart using the multipatch module. PLoS computational biology 2015;11(7):e1004284.

[6] Huntjens PR, Walmsley J, Ploux S, Bordachar P, Prinzen FW, Delhaas T, Lumens J. Influence of left ventricular lead position relative to scar location on response to cardiac resynchronization therapy: a model study. Europace 2014; 16(suppl_4):iv62-iv68.

[7] van Everdingen W, Walmsley J, Maarten C, De Boeck B, Meine M, Prinzen F, Delhaas T, Lumens J, Leenders G. Echocardiographic prediction of cardiac resynchronization therapy response requires analysis of both mechanical dyssynchrony and right ventricular function: A combined analysis of patient data and computer simulations. Journal of the American Society of Echocardiography 2017;

[8] Arts T, Reesink K, Kroon W, Delhaas T. Simulation of adaptation of blood vessel geometry to flow and pressure: Implications for arterio-venous impedance. Mechanics Research Communications 2012;42:15-21.

[9] Stolfo D, Tonet E, Merlo M, Barbati G, Gigli M, Pinamonti B, Ramani F, Zecchin M, Sinagra G. Early right ventricular response to cardiac resynchronization therapy: impact on clinical outcomes. European journal of heart failure 2016; 18(2):205-213.

Address for correspondence:

Clemens Zeile

Fakultaet fuer Mathematik, Universitaet Magdeburg, Universitaetsplatz 2, 39106 Magdeburg, Germany

clemens.zeile@ovgu.de 